\title{
WHEN CAN ONE DOMAIN ENCLOSE ANOTHER IN $\mathbb{R}^{3}$ ?
}

\author{
JIAZU ZHOU \\ (Received 2 February 1993; revised 1 July 1993) \\ Communicated by K. C. H. Mackenzie
}

\begin{abstract}
In this paper, we give a sufficient condition (Theorem) in order that one domain $D_{1}$ bounded by a $C^{2}$-smooth boundary can be enclosed in, or enclose, another domain $D_{0}$ bounded by the same kind of boundary. A same kind of sufficient condition for convex bodies (Corollary) is also obtained.

1991 Mathematics subject classification (Amer. Math. Soc.): primary 52A22, 53C21; secondary 51 M16. Keywords and phrases: Domain, convex body, curvature, mean curvature, kinematic measure, kinematic formula, quermassintegrals, circumscribed ball.
\end{abstract}

\section{Introduction}

Many mathematicians have been interested in getting sufficient conditions to insure that a given domain $D_{1}$ of surface area $F_{1}$, bounded by a piecewise smooth boundary $\partial D_{1}$, of volume $V_{1}$ may be moved 'inside' another domain $D_{0}$ of surface area $F_{0}$, bounded by a piecewise smooth boundary $\partial D_{0}$, of volume $V_{0}$. The general principle underlying this investigation can be briefly described as follows.

Let $D_{0}, D_{1}$ be two suitable domains in Euclidean space $\mathbb{R}^{n}$, for example, two convex bodies with interior points. Let $G$ be the group of rigid motions of $\mathbb{R}^{n}$ and let $m$ be its (suitably normalized) invariant measure. Then

(1) $m\left\{g \in G: g D_{1} \subset D_{0}\right.$ or $\left.g D_{1} \supset D_{0}\right\}$

$$
=m\left\{g \in G: D_{0} \cap g D_{1} \neq \emptyset\right\}-m\left\{g \in G: \partial D_{0} \cap g \partial D_{1} \neq \emptyset\right\} \text {. }
$$

(If $D_{0}, D_{1}$ are not convex, one assumes that their boundaries are connected.) By integral geometric methods it is possible to estimate the measure $m\left\{g \in G: D_{0} \cap\right.$ $\left.g D_{1} \neq \emptyset\right\}$ from below and the measure $m\left\{g \in G: \partial D_{0} \cap g \partial D_{1} \neq \emptyset\right\}$ from above in

(C) 1995 Australian Mathematical Society $0263-6115 / 95 \$ A 2.00+0.00$ 
terms of geometric invariants of $D_{0}$ and $D_{1}$. This results in an inequality of the form

$$
m\left\{g \in G: g D_{1} \subset D_{0} \text { or } g D_{1} \supset D_{0}\right\} \geq f\left(A_{0}^{1}, \ldots, A_{0}^{k} ; A_{1}^{1}, \ldots, A_{1}^{k}\right),
$$

where $A_{i}^{j}$ is a geometric invariant of $D_{i}(i=0,1)$, for example, volume, surface area, total mean curvature of the boundary $\partial D_{i}$, etcetera. One can then state the following conclusion: If $f\left(A_{0}^{1}, \ldots, A_{0}^{k} ; A_{1}^{1}, \ldots, A_{1}^{k}\right)>0$, then there is a rigid motion $g$ such that either $g D_{1}$ is contained in $D_{0}$ or $g D_{1}$ contains $D_{0}$.

In 1941 Hadwiger (see [9]) was the first to use the method of integral geometry to obtain some sufficient conditions in the Euclidean plane $\mathbb{R}^{2}$. Delin Ren (see [8]) in 1986 obtained other sufficient conditions in $\mathbb{R}^{2}$. But there was no general result or analogue of Hadwiger's theorem in Euclidian space $\mathbb{R}^{n}(n \geq 3)$ until the works $[11,12,13,14,15]$ appeared, even if some very strong restrictions are put on the domains involved. (For example, the domains are supposed to be convex bodies and some topological conditions are put on their boundaries and intersection.) The situation of $n$-dimensional space $\mathbb{R}^{n}(n \geq 3)$ is much more complex and difficult than that of the 2-dimensional plane $\mathbb{R}^{2}$. All the formulas and method in $\mathbb{R}^{2}$ cannot be directly transferred. Moreover, the situations and techniques appropriate to $\mathbb{R}^{3}$ and $\mathbb{R}^{2 k}(k \geq 2)$ are totally different due to different topological structures.

In this paper we try to obtain other analogues of Hadwiger's theorem in the space $\mathbb{R}^{3}$. We follow the ideas in [15] and estimate the arc length of the intersection curve $\partial D_{0} \cap g \partial D_{1}$ of the boundaries $\partial D_{0}, \partial D_{1}$ of two domains $D_{0}, D_{1}$ in $\mathbb{R}^{3}$. By restricting the Euler-Poincaré characteristic $\chi\left(D_{0} \cap g D_{1}\right)$ of the intersection $\partial D_{0} \cap g \partial D_{1}$ to be at most a finite integer $N_{0}$ for each $g$, a rigid motion in $\mathbb{R}^{3}$, we obtain a sufficient condition (Theorem) to insure that one domain $D_{0}$ with smooth boundary $\partial D_{0}$ can contain, or be contained in, another domain $D_{1}$ with the same kind of boundary $\partial D_{1}$. This is a natural assumption: for example, when $D_{0}$ and $D_{1}$ are convex bodies, $\chi\left(D_{0} \cap g D_{1}\right) \leq N_{0}=1$. As an easy consequence of our theorem we obtain a sufficient condition (Corollary) for one convex body $D_{1}$ with smooth boundary to be contained in, or to contain, another convex body $D_{0}$ with the same kind of boundary. As one would expect, the conditions are inequalities involving volumes, surface areas and curvature integrals of the boundaries. Finally, we give an application of our geometric inequality.

\section{Main results and the proof}

For a $C^{2}$-smooth surface $\Sigma$ in Euclidean space $\mathbb{R}^{3}$, denote by $K$ its Gaussian curvature and by $H$ the mean curvature. Let $\tilde{K}, \tilde{H}$ and $\tilde{H}^{(2)}$, respectively, be the total Gaussian curvature, the total mean curvature and the total square mean curvature, 
that is

$$
\tilde{K}=\int_{\Sigma} K d \sigma, \quad \tilde{H}=\int_{\Sigma} H d \sigma, \quad \tilde{H}^{(2)}=\int_{\Sigma} H^{2} d \sigma,
$$

where $d \sigma$ is the volume element of $\Sigma$.

In this paper, we suppose that the domains $D_{i}(i=0,1)$ in $\mathbb{R}^{3}$ are bounded by $C^{2}$-smooth surfaces $\partial D_{i}$. Denote by $V_{i}$ the volumes and by $F_{i}$ the surface areas, respectively. Let $\tilde{K}_{i}, \tilde{H}_{i}$ and $\tilde{H}_{i}^{(2)}$ be the total Gaussian curvature, the total mean curvature and the total square mean curvature of $\partial D_{i}$, respectively. Denote by $\chi(\cdot)$ the Euler-Poincaré characteristic. We have the following conclusion:

THEOREM. Let $D_{i}(i=0,1)$ be domains in $\mathbb{R}^{3}$ with connected $C^{2}$-smooth boundaries $\partial D_{i}$ such that for all $g \in G$, the group of rigid motions in $\mathbb{R}^{3}$, the EulerPoincaré characteristic $\chi\left(D_{0} \cap g D_{1}\right)$ of the intersection $\partial D_{0} \cap g \partial D_{1}$, satisfies $\chi\left(D_{0} \cap g D_{1}\right) \leq N_{0}$, a finite integer. Then a sufficient condition for $D_{1}$ to be contained in, or to contain, $D_{0}$ is

$$
\begin{aligned}
& 8 \pi\left(V_{0} \chi\left(D_{1}\right)+V_{1} \chi\left(D_{0}\right)\right)+2\left(F_{0} \tilde{H}_{1}+F_{1} \tilde{H}_{0}\right) \\
& \quad-N_{0} \cdot \pi R\left[3\left(F_{0} \tilde{H}_{1}^{(2)}+F_{1} \tilde{H}_{0}^{(2)}\right)-2 \pi\left(F_{0} \chi\left(\partial D_{1}\right)+F_{1} \chi\left(\partial D_{0}\right)\right)\right]>0
\end{aligned}
$$

where $R$ is the smaller radius of the circumscribed balls of $D_{0}$ and $D_{1}$. Moreover,

(i) if $V_{1} \geq V_{0}$, then $D_{0}$ can be contained in $D_{1}$;

(ii) if $V_{0} \geq V_{1}$, then $D_{0}$ can contain $D_{1}$.

Consider two domains $D_{i}(i=0,1)$ with connected $C^{2}$-smooth boundaries $\partial D_{i}$ in $\mathbb{R}^{3}$, one fixed and the other moving under the group $G$ of rigid motions in $\mathbb{R}^{3}$. Let the fixed one be $D_{0}$ and the moving one be $g D_{1}$ for $g \in G$, and let $d g$ be the kinematic density so normalized that the measure of all position about a point is $8 \pi^{2}$. Then we have C-S. Chen's kinematic formula first proved (see [3]) in 1972 and then reproved (see [15]) by this author in 1991 by a different method:

(5) $\int_{\left\{g: \partial D_{0} \cap g \partial D_{1} \neq \emptyset\right\}}\left(\int_{C_{g}} \kappa_{C_{g}}^{2} d s\right) d g=2 \pi^{3}\left(3 \tilde{H}_{0}^{(2)}-\tilde{K}_{0}\right) F_{1}+2 \pi^{3}\left(3 \tilde{H}_{1}^{(2)}-\tilde{K}_{1}\right) F_{0}$,

where $\kappa_{C_{g}}$ is the curvature of the intersection curve $C_{g}=\partial D_{0} \cap g \partial D_{1}$ and $d s$ is the arc element. Generically, the intersection curve $C_{g}$ is composed of several components, that is, several simple closed curves. For every rigid motion $g \in G$, let $C_{g}=\partial D_{0} \cap g \partial D_{1}=\bigcup_{i}^{N_{R}} C_{i}$, where $N_{g}$ is finite for almost all $g$.

For a $C^{2}$-smooth simple closed curve $C$ in $\mathbb{R}^{3}$, we have the inequality (see [1])

$$
L \leq R_{C} \int_{C} \kappa_{C} d s
$$


where $L$ is the length of curve $C, d s$ is the arc element, $\kappa_{C}$ the curvature of $C$ and $R_{C}$ the radius of the circumscribed ball of $C$.

The kinematic fundamental formula of Blaschke (see $[9,4]$ ) reads

(7) $\int_{\left\{g: D_{0} \cap g D_{1} \neq \emptyset\right]} \chi\left(D_{0} \cap g D_{1}\right) d g=8 \pi^{2}\left(V_{0} \chi\left(D_{1}\right)+V_{1} \chi\left(D_{0}\right)\right)+2 \pi\left(F_{0} \tilde{H}_{1}+F_{1} \tilde{H}_{0}\right)$.

PROOF OF THEOREM. By Hölder's inequality and (6) we have

$$
\begin{aligned}
& \left(\int_{C_{R}} \kappa_{C_{g}} d s\right)^{2} \leq\left(\int_{C_{g}} 1^{2} \cdot d s\right)\left(\int_{C_{g}} \kappa_{C_{g}}^{2} d s\right) \\
& =\left(\sum_{i}^{N_{g}} L_{i}\right)\left(\int_{C_{g}} \kappa_{C_{g}}^{2} d s\right) \leq\left(\sum_{i}^{N_{g}} R_{C_{i}} \int_{C_{i}} \kappa_{C_{i}} d s\right)\left(\int_{C_{g}} \kappa_{C_{g}}^{2} d s\right) \\
& \leq R_{g}\left(\sum_{i}^{N_{g}} \int_{C_{i}} \kappa_{C_{i}} d s\right)\left(\int_{C_{g}} \kappa_{C_{g}}^{2} d s\right)=R_{g}\left(\int_{C_{g}} \kappa_{C_{g}} d s\right)\left(\int_{C_{g}} \kappa_{C_{g}}^{2} d s\right),
\end{aligned}
$$

where $R_{C_{i}}$ is the radius of the circumscribed ball of $C_{i}$, and $R_{g}=\max \left\{R_{C_{1}}, \cdots, R_{C_{N_{g}}}\right\}$.

Fenchel's theorem reads

$$
\int_{C} \kappa_{C} d s \geq 2 \pi
$$

where $C$ is a simple closed curve, with equality holding if and only if $C$ is a plane convex curve. Using this in (8) gives

$$
2 \pi \leq \int_{C_{g}} \kappa_{C_{g}} d s \leq R_{g} \int_{C_{g}} \kappa_{C_{g}}^{2} d s \leq R \int_{C_{k}} \kappa_{C_{g}}^{2} d s,
$$

where $R$ is the smaller radius of the circumscribed balls of $D_{0}$ and $D_{1}$. For a fixed $g \in G$, equality in

$$
2 \pi \leq \int_{C_{g}} \kappa_{C_{g}} d s \leq R_{g} \int_{C_{g}} \kappa_{C_{g}}^{2} d s
$$

holds if and only if $C_{g}$ is a circle. If for almost all $g \in G$ the equality in

$$
2 \pi \leq \int_{C_{g}} \kappa_{C_{g}} d s
$$

holds, then all $C_{g}$ must be plane convex curves (Fenchel). This will force $D_{0}$ and $D_{1}$ to be two balls - a result due to Goodey, see $[6,5]$.

If we integrate the inequality $2 \pi \leq R \int_{C_{g}} \kappa_{C_{g}}^{2} d s$ over $\left\{g: \partial D_{0} \cap g \partial D_{1} \neq \emptyset\right\}$, then by (5) we obtain 


$$
\begin{aligned}
\int_{\left\{g: \partial D_{0} \cap g \partial D_{1} \neq \emptyset\right\}} d g & \leq \frac{R}{2 \pi} \int_{\left\{g: \partial D_{0} \cap g \partial D_{1} \neq \emptyset\right\}}\left(\int_{C_{g}} \kappa_{C_{g}}^{2} d s\right) d g \\
& =\pi^{2} R\left[\left(3 \tilde{H}_{0}^{(2)}-\tilde{K}_{0}\right) F_{1}+\left(3 \tilde{H}_{1}^{(2)}-\tilde{K}_{1}\right) F_{0}\right] .
\end{aligned}
$$

By our supposition on $D_{0}$ and $D_{1}$ we have

$$
\int_{\left\{g: D_{0} \cap g D_{1} \neq \emptyset\right\}} \chi\left(D_{0} \cap g D_{1}\right) d g \leq N_{0} \int_{\left\{g: D_{0} \cap g D_{1} \neq \emptyset\right\}} d g .
$$

From (7), (13) and (14) we have the kinematic measure of one domain moving to another under the group $G$ of rigid motions in $\mathbb{R}^{3}$, that is,

$$
\begin{aligned}
& m\left\{g \in G: g D_{1} \subseteq D_{0} \quad \text { or } \quad g D_{0} \subseteq D_{1}\right\} \\
& =\int_{\left\{g: g D_{1} \subseteq D_{0} \text { or } g D_{0} \subseteq D_{1}\right\}} d g \\
& =\int_{\left\{g: D_{0} \cap g D_{1} \neq \emptyset\right\}} d g-\int_{\left[g: \partial D_{0} \cap g \partial D_{1} \neq \emptyset\right]} d g \\
& \geq \frac{1}{N_{0}}\left[8 \pi^{2}\left(V_{0} \chi\left(D_{1}\right)+V_{1} \chi\left(D_{0}\right)\right)+2 \pi\left(F_{0} \tilde{H}_{1}+F_{1} \tilde{H}_{0}\right)\right] \\
& -\pi^{2} R\left[3\left(F_{0} \tilde{H}_{1}^{(2)}+F_{1} \tilde{H}_{0}^{(2)}\right)-\left(F_{0} \tilde{K}_{1}+F_{1} \tilde{K}_{0}\right)\right] \\
& =\frac{1}{N_{0}}\left[8 \pi^{2}\left(V_{0} \chi\left(D_{1}\right)+V_{1} \chi\left(D_{0}\right)\right)+2 \pi\left(F_{0} \tilde{H}_{1}+F_{1} \tilde{H}_{0}\right)\right] \\
& -\pi^{2} R\left[3\left(F_{0} \tilde{H}_{1}^{(2)}+F_{1} \tilde{H}_{0}^{(2)}\right)-2 \pi\left(F_{0} \chi\left(\partial D_{1}\right)+F_{1} \chi\left(\partial D_{0}\right)\right)\right] .
\end{aligned}
$$

The last equality comes from the Gauss-Bonnet formula $\tilde{K}_{i}=2 \pi \cdot \chi\left(\partial D_{i}\right)(i=0,1)$. This proves the theorem.

If $D_{0}$ and $D_{1}$ are convex bodies in $\mathbb{R}^{3}$, we have $\chi\left(D_{0}\right)=\chi\left(D_{1}\right)=N_{0}=1$. Denote by $W_{2}^{i}$ the quermassintegrals [9] of the convex bodies $D_{i}$. Then we have the following consequence.

COROLLARY. Let $D_{i}(i=0,1)$ be two convex bodies in $\mathbb{R}^{3}$ with $C^{2}$-smooth boundaries $\partial D_{i}$, and denote by $\tilde{H}_{i}^{(2)}$ the total square of the mean curvatures of $\partial D_{i}$. Then a sufficient condition for a convex body $D_{0}$ to contain, or to be contained in, another convex body $D_{1}$, is

(16) $8 \pi\left(V_{0}+V_{1}\right)+6\left(F_{0} W_{2}^{1}+F_{1} W_{2}^{0}\right)-\pi R\left[3\left(F_{0} \tilde{H}_{1}^{(2)}+F_{1} \tilde{H}_{0}^{(2)}\right)-4 \pi\left(F_{0}+F_{1}\right)\right]>0$.

Moreover,

(i) if $V_{1} \geq V_{0}$, then $D_{0}$ can be contained in $D_{1}$;

(ii) if $V_{0} \geq V_{1}$, then $D_{0}$ can contain $D_{1}$. 


\section{Remarks}

REMARK 1. Let $D_{1}$ be a convex body with diameter $2 R$, and let $D_{0}$ be a ball of radius $R$. Since we have neither $D_{0} \subset D_{1}$ nor $D_{1} \subset D_{0}$, the condition (16) cannot hold. Thus we have

$$
\begin{aligned}
& 8 \pi\left(V_{0}+V_{1}\right)+6\left(F_{0} W_{2}^{1}+F_{1} W_{2}^{0}\right) \\
& \quad-\frac{\pi \delta_{m}}{2}\left[3\left(F_{0} \tilde{H}_{1}^{(2)}+F_{1} \tilde{H}_{0}^{(2)}\right)-4 \pi\left(F_{0}+F_{1}\right)\right] \leq 0,
\end{aligned}
$$

that is,

$$
\tilde{H}_{1}^{(2)} \geq \frac{4}{9}(2+3 \pi)+\frac{2(1-\pi)}{3 R^{2} \pi} F_{1}+\frac{2}{3 R^{3} \pi} V_{1}+\frac{2}{R \pi} W_{2}^{1} .
$$

Formula (18) is an application of our formula (16), that is:

Let $\Sigma$ be a convex surface in $\mathbb{R}^{3}$ and $2 R$ its diameter. Denote by d $\sigma, F, V, W$ and $H$ the volume element, surface area, the volume bounded, the quermassintegral of the convex body bounded by $\Sigma$, and mean curvature, respectively. Then we have the inequality

$$
\int_{\Sigma} H^{2} d \sigma \geq \frac{4}{9}(2+3 \pi)+\frac{2}{R \pi} W+\frac{2(1-\pi)}{3 R^{2} \pi} F+\frac{2}{3 R^{3} \pi} V .
$$

Note that the right-hand side of inequality (19) is well-defined for any convex body $D$, whereas the definition of $H$ and the integral $\int_{\partial D} H^{2} d \sigma$ makes sense only if $\partial D$ is of class $C^{2}$. But by inequality (19), for a convex body with piecewise $C^{2}$-smooth boundary $\partial D$, we can estimate the integral $\int_{\partial D} H^{2} d \sigma$.

B.-Y. Chen [2] and others give the following estimate:

$$
\int_{\Sigma} H^{2} d \sigma \geq 4 \pi
$$

One would see that (19) can give the estimates which are much bigger than $4 \pi$ for some convex surfaces.

REMARK 2. It would be interesting to remove the 'smoothness' restriction on the convex bodies involved in the corollary. All the notions except $\tilde{H}^{(2)}$ here are welldefined for non-smooth convex bodies. If we could find a substitute for $\tilde{H}^{2}$, the consequences in this paper could be interpreted for arbitrary convex bodies. This is definitely worth further investigation.

REMARK 3. Of course, these conditions are not necessary. 


\section{Acknowledgement}

I would like to thank Eric Grinberg and Delin Ren for some discussions, support and encouragement. I would also like to thank E. Lutwak, P. R. Goodey and R. Howard for their interest and comments. Finally, I thank the referee for some suggestions.

\section{References}

[1] Yu. D. Burago 1. A. Zalgaller, Geometric inequalities (Springer, Berlin, 1988).

[2] B-Y Chen, Geometry of submanifolds (Marcel Dekker, New York, 1973).

[3] C-S. Chen, 'On the kinematic formula of square of mean curvature', Indiana Univ. Math. J. 22 (1972-73), 1163-1169.

[4] S. S. Chern, 'On the kinematic formula in the euclidean space of n dimensions', Amer. J. Math. 74 (1952), 227-236.

[5] P. Goodey, 'Homothetic ellipsoids', Math. Proc. Cambridge Philos. Soc. 93 (1983), 25-34.

[6] P. R. Goodey, 'Connectivity and free rolling convex bodies', Mathematika 29 (1982), 249-259.

[7] R. Howard, 'The kinematic formula in riemannian geometry', Mem. Amer. Math. Soc., to appear.

[8] Delin Ren, Introduction to integral geometry (Shanghai Press of Sciences and Technology, Shanghai, 1987).

[9] L. A. Santaló, Integral geometry and geometric probability (Addision-Wesley, Reading, 1976).

[10] Michael Spivak, A comprehensive introduction to differential geometry (II) (Publish or Perish, Houston, 1979).

[11] Gaoyong Zhang, 'A sufficient condition for one convex body containing another', Chinese Ann. Math. Ser. B 9 (1988), 447-451.

[12] Jiazu Zhou, 'Analogues of Hadwiger's theorem-sufficient conditions for one convex body to fit another in $\mathbb{R}^{3}$, preprint.

[13] _ , 'The sufficient condition for a convex body to fit another in $\mathbb{R}^{4}$ ', Proc. Amer. Math. Soc. 121 (1994), 907-913.

[14] - 'Analogues of Hadwiger's theorem in space $\mathbb{R}^{n}$ and sufficient condition for a convex domain to enclose another', Acta. Math. Sinica, to appear.

[15] , 'A kinematic formula and analogues of Hadwiger's theorem in space', Contemp. Math. 140 (1992), 159-167.

[16] _ 'Kinematic formulas for total square of mean curvature of hypersurfaces', Bull. Inst. Math. Acad. Sinica. 22 (1994), 31-47.

Department of Mathematics

Temple University

Philadelphia, PA 19122

USA

e-mail: zhou@euclid.math.temple.edu 